\title{
The Origin of Organic Matter in the Solar System: Evidence from the Interplanetary Dust Particles
}

\author{
G. J. Flynn \\ Dept. of Physics, SUNY-Plattsburgh, 101 Broad St, Plattsburgh, NY \\ 12901 USA \\ L. P. Keller
}

NASA Johnson Space Center, Houston, TX 77051 USA

C. Jacobsen \& S. Wirick

Dept. of Physics, SUNY-Stony Brook, Stony Brook, NY 11794

\begin{abstract}
Interplanetary dust particles (IDPs), $\sim 10 \mu \mathrm{m}$ particles from comets and asteroids, have been collected by NASA from the Earth's stratosphere. We compared carbon X-ray Absorption Near-Edge Structure (XANES) and Fourier Transform Infra-Red (FTIR) spectra of anhydrous and hydrated interplanetary dust particles and found that anhydrous and hydrated IDPs have similar types and abundances of organic carbon. This is different from results on meteorites, which show that hydrated carbonaceous meteorites contain abundant organic matter, while anhydrous carbonaceous meteorites contain less carbon mostly in elemental form. But all anhydrous carbonaceous meteorites are depleted in moderately volatile and volatile elements in a pattern that suggested they experienced temperatures in excess of $1200^{\circ} \mathrm{C}$, a temperature sufficient to destroy any organic matter they originally contained, while many anhydrous IDPs show no evidence of severe heating. These IDP results indicate that the bulk of the pre-biotic organic matter in extraterrestrial materials formed before aqueous processing, possibly by irradiation of C-bearing ices or by a Fisher-Tropsch type process operating in the gas phase of the nebula or in the interstellar medium.
\end{abstract}

\section{Introduction}

The origin of the organic matter in interplanetary materials has not been established. A variety of production mechanisms have been proposed, with two extreme cases being a Fisher-Tropsch type process operating in the gas phase of the solar nebula (or possibly in the interstellar medium) or a Miller-Urey type process, which requires interaction with an aqueous fluid, presumably occurring on an asteroid (Cronin, Pizzarello \& Cruikshank 1988). In the Fisher-Tropsch case, we might expect similar organic matter in hydrated and anhydrous interplanetary materials. However, aqueous alteration is required in the case of 
the Miller-Urey process, and we would expect to see organic matter preferentially associated with interplanetary materials that exhibit evidence of aqueous activity, such as the presence of hydrated silicates.

The types and abundance of organic matter in meteorites have been used as an indicator of the origin of organic matter in the Solar System. Indigenous complex organic matter, including amino acids, has been found in hydrated carbonaceous chondrite meteorites, such as Murchison. Much lower amounts of complex organic matter, possibly only terrestrial contamination, have been found in anhydrous carbonaceous chondrite meteorites, such as Allende, that contain most of their carbon in elemental form (Cronin, Pizzarello \& Cruikshank 1988). These results seem to favor production of the bulk of the pre-biotic organic matter in the Solar System by aqueous processing on parent bodies such as asteroids, a Miller-Urey type of process.

However, the hydrated carbonaceous chondrite meteorites have approximately solar abundances of the moderately volatile elements, while all anhydrous carbonaceous chondrite meteorites have significantly lower contents of these moderately volatile elements. Two mechanisms, incomplete condensation or evaporation, both of which involve processing at $\sim 1200^{\circ} \mathrm{C}$ or higher, have been suggested to explain the lower content of the moderately volatile elements in all anhydrous meteorites (Palme 2000). The high temperatures associated with either the incomplete condensation or the subsequent vaporization are sufficient to remove or destroy most organic matter in the anhydrous meteorites, indicating that the meteorite studies do not constrain the origin of organic matter.

\section{Samples Analyzed}

Most anhydrous interplanetary dust particles (IDPs), dust from comets and asteroids collected from the Earth's stratosphere, have volatile contents higher than the hydrated carbonaceous meteorites, suggesting these IDPs experienced minimal thermal processing. Unequilibrated mineralogy indicates many IDPs never experienced significant heating. Some IDPs have regions with high $D / H$ within a few $\mu \mathrm{m}$ of other phases with lower $D / H$, indicating these IDPs were not heated to the point of $D$ equilibration since IDP formation. Thus, these anhydrous IDPs are more primitive than any anhydrous meteorite.

To infer the origin of organic matter in the Solar System we measured the abundance and types of carbon in all three kinds of primitive (i.e., not thermally processed) extraterrestrial materials available for laboratory analysis: anhydrous IDPs, hydrated IDPs, and hydrated carbonaceous chondrite meteorites.

\section{Analysis Techniques}

Because of the small sizes $(\sim 10 \mu \mathrm{m})$ of the IDPs, the organic carbon in IDPs cannot be characterized by the traditional analysis techniques. We employ two synchrotron-based instruments at the National Synchrotron Light Source (Brookhaven National Laboratory):

1. A Scanning Transmission X-ray Microscope (STXM), to map the carbon distribution and determine the carbon abundance, and to perform Carbon 
X-ray Absorption Near Edge Structure (XANES) spectroscopy, to identify the carbon functional groups in the particles, and,

2. A Fourier Transform Infrared (FTIR) spectrometer, to detect and identify the functional groups of organic compounds in the particles.

The analysis methods and results are described in Flynn et al. (2000).

\section{STXM Results}

STXM carbon maps of 12 IDPs indicate they have carbon contents ranging from a few up to 90 vol.-\%. The carbon contents are not substantially different in the hydrated and anhydrous subgroups, though all the IDPs having the highest carbon contents are anhydrous. The 3 hydrated IDPs and 7 of the 9 anhydrous IDPs we examined using the STXM have essentially identical C-XANES spectra (see Fig. 1), and these spectra are generally similar to the C-XANES spectrum of acid-insoluble organic matter extracted from the Murchison (CM2) meteorite. Each of these C-XANES spectra shows a large absorption at $\sim 285 \mathrm{eV}$, characteristic of the C-ring structure, and a second strong absorption at $\sim 288.5 \mathrm{eV}$, characteristic of the $\mathrm{C}=\mathrm{O}$ bond (and distinct from the $\sim 290 \mathrm{eV}$ absorption of the $\mathrm{C}-\mathrm{O}$ in carbonate). The high carbonyl abundance indicates that a substantial fraction of the carbon in the IDPs is organic rather than elemental (Flynn et al. 2000).

If the organic matter in these samples is non-crystalline, then the ratio of the area in the $\mathrm{C}$-ring absorption to the area in the $\mathrm{C}=\mathrm{O}$ absorption measures the $\mathrm{C}$-ring to $\mathrm{C}=\mathrm{O}$ functional groups. Thus, these results indicate that the anhydrous IDPs and the hydrated meteorites both contain organic matter with very similar ratios of $\mathrm{C}$-ring to $\mathrm{C}=\mathrm{O}$ (carbonyl) functional group. However, the C-ring/C=O ratio appears to be somewhat higher in Murchison than in either the anhydrous or the hydrated IDPs.

\section{FTIR Results}

The IDPs are dominated by anhydrous and hydrated silicates, carbonates, sulfides, and oxides, which have strong absorptions over most of the 4000 to $650 \mathrm{~cm}^{-1}$ range we analyze by FTIR. This interferes with the detection of organic features over much of the IR spectrum. However, these phases do not interfere from 2700 to $3100 \mathrm{~cm}^{-1}$, where $\mathrm{C}-\mathrm{H}$ stretching vibrations occur. So, we have focused our efforts on examining this region of the IR spectrum.

Eight of the 11 IDPs examined by FTIR showed strong $\mathrm{C}-\mathrm{H}_{2}$ and $\mathrm{C}-\mathrm{H}_{3}$ stretching vibrations of aliphatic hydrocarbon, as shown in Fig. 2. The 2700 to $3100 \mathrm{~cm}^{-1}$ region of the spectra of hydrated and of anhydrous IDPs were very similar, and were also similar to the spectrum of bulk Murchison.

The ratio of the depth of the $\mathrm{C}-\mathrm{H}_{2}$ absorption to the $\mathrm{C}-\mathrm{H}_{3}$ absorption is a measure of the mean length of the aliphatic hydrocarbon. The similarity of the three spectra, shown in Fig. 2, indicates that the anhydrous IDPs, the hydrated IDPs, and the hydrated meteorites all contain an aliphatic organic compound (or mixture of compounds) having approximately the same aliphatic chain length. 


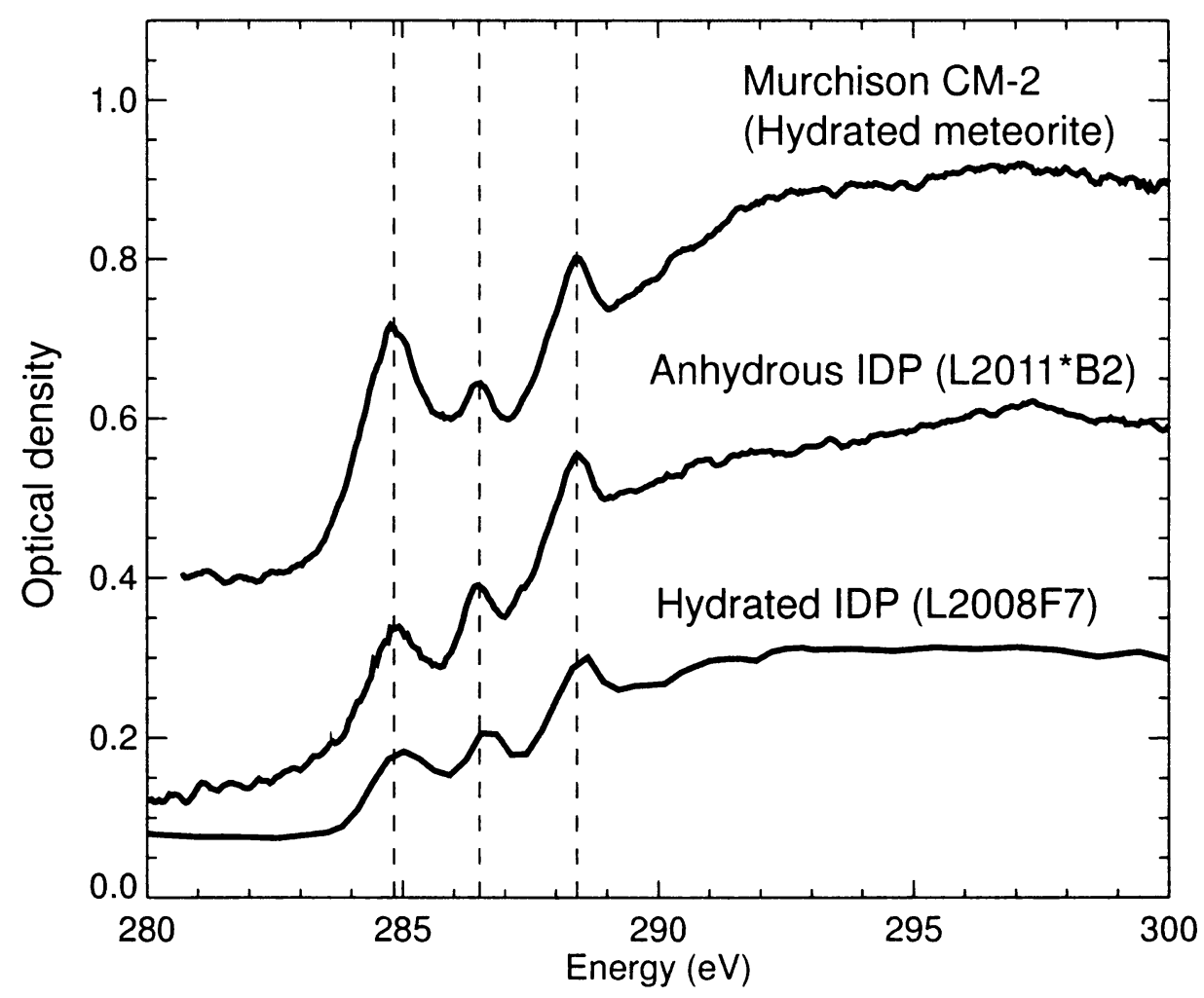

Figure 1. Comparison of the C-XANES spectra of the acid insoluble residue from the Murchison meteorite, an anhydrous IDP, and a hydrated IDP. The apparent small energy shift $(\sim 0.1 \mathrm{eV})$ in the hydrated IDP spectrum compared to the other two spectra is comparable to the STXM energy resolution, and may have resulted from a small shift in the monochrometer energy for that measurement

There are small variations in the depth of the $\mathrm{C}-\mathrm{H}_{3}$ to $\mathrm{C}-\mathrm{H}_{2}$ ratio from sample to sample, but we see variations of approximately the same magnitude from IDP to IDP.

Brownlee has developed an acid-etching technique for IDPs, similar to the technique for preparing the acid insoluble kerogen extract from meteorites. This acid-etching technique removes the silicate, carbonate, and sulfide minerals, which interfere with most organic absorption features, from the IDPs. The acid etching leaves most of the carbonaceous matter (and probably some insoluble oxides). The absence of silicate, carbonate, and sulfide allows us to compare the full IR spectra, over the range from 4000 to $650 \mathrm{~cm}^{-1}$, of anhydrous and hydrated IDPs to the spectra of acid insoluble extract from hydrated meteorites. We performed FTIR analysis on two acid etched IDPs, one anhydrous and one hydrated (Flynn et al. 2002). We detected the carbonyl absorption near $1710 \mathrm{~cm}^{-1}$, confirming the $\mathrm{C}=\mathrm{O}$ identification by $\mathrm{C}-\mathrm{XANES}$, and showing that the carbonyl is dominantly bonded in aliphatic ketones. Further, we found 


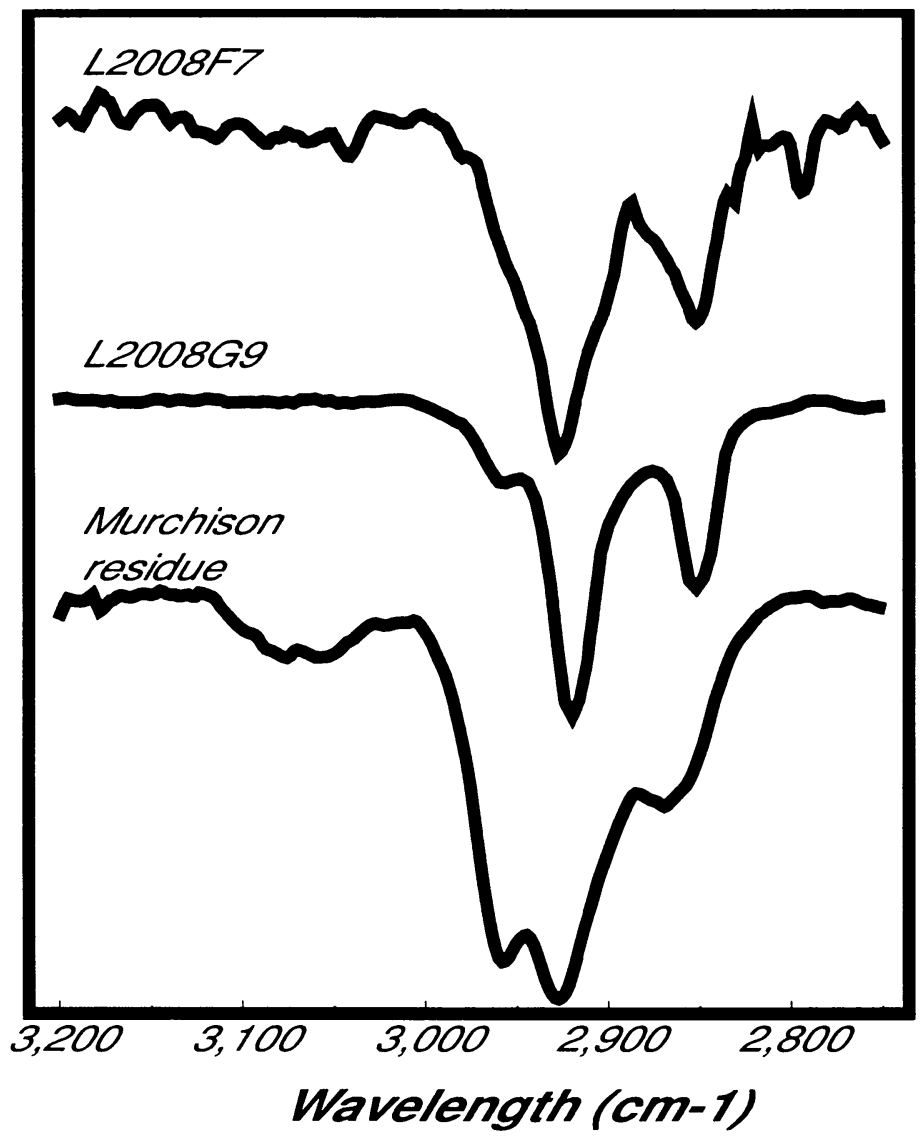

Figure 2. Comparison of the 3 micron $\mathrm{C}-\mathrm{H}$ stretching region infrared spectra of Murchison matrix, an anhydrous IDP and a hydrated IDP

similar ratios of the $\mathrm{C}-\mathrm{H}$ absorption features to the $\mathrm{C}=\mathrm{O}$ absorption feature in the two types of IDPs, again showing that the bulk of the organic matter is similar in anhydrous and hydrated IDPs.

\section{Conclusions}

The similarity of the $\mathrm{C}$ abundance, the C-XANES and the FTIR spectra of anhydrous IDPs, hydrated IDPs, and hydrated carbonaceous meteorites are /emphconsistent with the production of much of the pre-biotic organic matter in the Solar System prior to aqueous alteration. Some of the carbonaceous material in IDPs formes the "glue" that holds the silicate crystals together to form the paricles. This suggests the organic matter formed prior to dust aggregation, possibly by irradiation of C-bearing ices or by a Fisher-Tropsch type process operating in the gas phase of the nebula or in the interstellar medium (given large D excesses reported in some IDPs). The small differences between Murchi- 
son and the anhydrous IDPs - an apparently higher C-ring/C-O ratio and an apparently higher $\mathrm{C}-\mathrm{H}_{3}$ to $\mathrm{C}-\mathrm{H}_{2}$ ratio in Murchison - need to be investigated in more detail.

\section{References}

Cronin, J. R., Pizzarello, S., \& Cruikshank D. P. 1988, in Meteorites and the Early Solar System, ed. J. F. Kerridge \& M. S. Matthews, (University of Arizona Press), 819

Flynn G. J., Keller, L. P., Jacobsen, C., Wirick, S., \& Miller, M. A. 2000, in ASP Conf. Ser. 213, Bioastronomy '99: A New Era in the Search for Life, ed. G. Lemarchand \& K. Meech, (Astron. Soc. of the Pacific Press), 191

Flynn, G. J., Keller, L. P., Joswiak, D., \& Brownlee, D. E. 2002, in Lunar \& Planetary Science XXXIII, Lunar \& Planetary Institute, Houston TX, CD-ROM, Abs. \# 1320

Palme, H. 2000, in From Dust to Terrestrial Planets, ed. W. Benz, R. Kallenbach, \& G. W. Lugmair (Boston: Kluwer Academic Publishers) 237

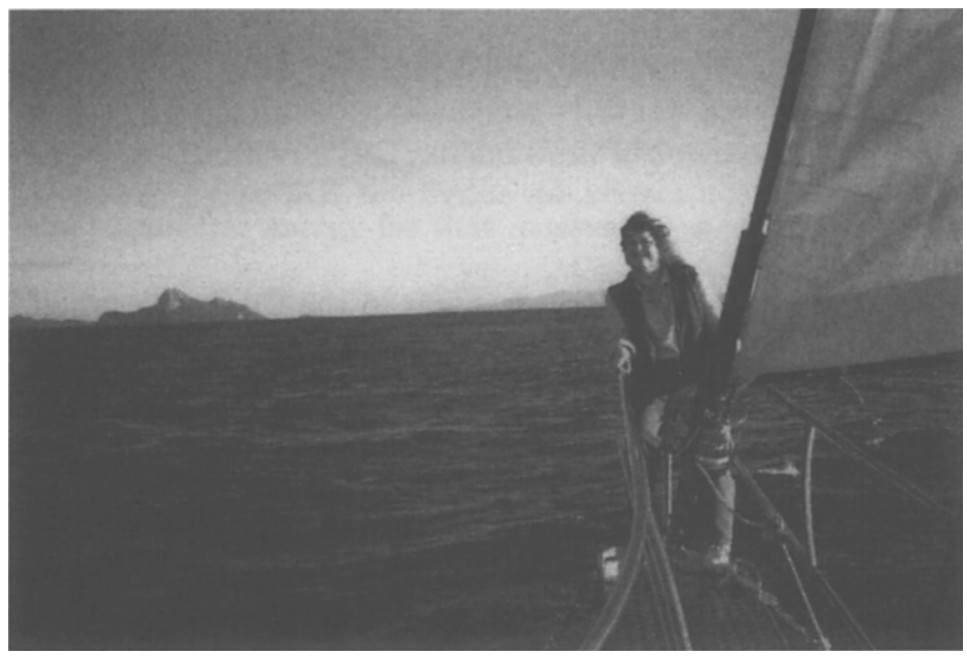

(photo: Ray Norris) 